\title{
Predictors of functional outcome in patients with stroke thrombolysis in a tertiary hospital in Malaysia
}

\author{
Mei-Ling Sharon $\underline{T a i}^{1}$, MBBS, MRCP, Khean Jin $\underline{G o h}^{1}$, MBBS, FRCP, Khairul Azmi Abdul Kadir ${ }^{2}$, MBBS, MMed, \\ Mohd Idzwan Zakaria ${ }^{3}$, MB BCh BAO, MMed, Jun Fai $\underline{\text { Yap }}^{1}$, MBBS, Kay Sin $\underline{\operatorname{Tan}}^{1}$, MBBS, FRCP
}

INTRODUCTION Intravenous (IV) thrombolysis with alteplase (rt-PA) is effective in ischaemic stroke. The primary objective was to evaluate predictors of functional outcome in acute ischaemic stroke (AIS) patients treated with IV rt-PA. The secondary objective was to assess the outcome with the modified Rankin scale (mRS). We also examined the predictive value of the Totaled Health Risks in Vascular Events (THRIVE) score.

METHODS AIS patients treated with IV rt-PA from February 2012 to August 2016 were recruited. Demographic data, National Institutes of Health Stroke Scale (NIHSS) scores, timing and neuroradiological findings were recorded. Patients received a dose of $0.9 \mathrm{mg} / \mathrm{kg}$ IV rt-PA within 4.5 hours of symptom onset. mRS score was evaluated at discharge and three months, and good and poor clinical outcomes were defined as scores of 0-2 and 3-6, respectively. Baseline THRIVE scores were assessed.

RESULTS 36 patients received IV rt-PA. 20 (55.6\%) patients had an mRS score of 0-2 at three months. Based on THRIVE score, $86.1 \%$ had a good or moderately good prognosis. On univariate analysis, poor outcome was associated with NIHSS score before rt-PA $(p=0.03)$, THRIVE score $(p=0.02)$, stroke subtype $(p=0.049)$ and diabetes mellitus $(D M ; p=0.06)$. Multiple logistic regression showed that outcome was significantly associated with NIHSS score before rt-PA $(p=0.032)$ and DM $(p=0.010)$.

CONCLUSION Our newly developed Malaysian IV rt-PA service is safe, with similar outcomes to the published literature. Functional outcome after thrombolysis was associated with baseline NIHSS score and DM.

Keywords: functional, outcome, rt-PA, stroke, thrombolysis

\section{INTRODUCTION}

Stroke is a common disease worldwide and a leading cause of death worldwide. ${ }^{(1)}$ In Malaysia, stroke is the second most common cause of mortality and second leading cause of disease burden. ${ }^{(2,3)}$ In addition, it was the fifth most common cause of hospitalisation locally in 2015. ${ }^{(2,4)}$ Malaysia is a developing country in Southeast Asia with a multi-ethnic population of 28.3 million in 2010. ${ }^{(5)}$ Approximately $91.8 \%$ of the population are Malaysian citizens and $8.2 \%$ are non-citizens. ${ }^{(5)}$ The ethnic distribution of the country is as follows: Malays (63.1\%), Chinese (24.6\%), Indians (7.3\%) and others (5.0\%). ${ }^{(5)}$

Intravenous (IV) thrombolysis with alteplase (rt-PA) is effective in the management of acute ischaemic stroke (AIS) patients. ${ }^{(6)}$ The administration of rt-PA results in better functional outcome, usually measured by the modified Rankin scale (mRS). ${ }^{(6,7)}$ The number needed to treat for good functional outcome (mRS 0-1) when rt-PA is administered $<3$ hours from symptom onset is $11 .{ }^{(7)}$ Moreover, the number needed to treat for good outcome in the patients treated 3.0-4.5 hours from stroke onset is 20. ${ }^{(7)}$ The Totaled Health Risks in Vascular Events (THRIVE) score is a valuable, internationally validated tool in predicting the prognosis in Western patients with AIS after thrombolysis. ${ }^{(8)}$ A THRIVE score of $0-2$ is associated with a better prognosis, whereas a score of 6-9 is associated with a poorer prognosis. ${ }^{(9)}$
Our tertiary hospital started a stroke thrombolysis service in February 2012 and currently offers a 24-hour acute stroke thrombolysis service. However, to date, there has been no literature on the functional outcome of stroke patients administered with rt-PA in Malaysia. Hence, the primary objective of this study was to evaluate the predictors of functional outcome in Malaysian AIS patients who were treated with IV rt-PA. The secondary objective was to assess the clinical outcome of the thrombolysed stroke patients using the modified Rankin scale (mRS). We also aimed to examine the predictive value of THRIVE score in this thrombolysis cohort.

\section{METHODS}

This retrospective study was approved by the Institutional Ethics Committee of University of Malaya Medical Centre (UMMC), Kuala Lumpur, Malaysia. We recruited patients who were admitted to UMMC, Kuala Lumpur, Malaysia, for AIS between February 2012 and August 2016. The data of consecutive patients from the stroke thrombolysis registry was recorded.

The inclusion and exclusion criteria for stroke thrombolysis were based on the National Institute of Neurological Disorders and Stroke trial in the United States. ${ }^{(10)}$ The baseline demographic and clinical data of patients, including age, gender, ethnicity, vascular risk factors, stroke subtypes, blood pressure on admission, National Institutes of Health Stroke Scale (NIHSS)

${ }^{1}$ Division of Neurology, Department of Medicine, ${ }^{2}$ Department of Biomedical Imaging, ${ }^{3}$ Department of Emergency Medicine, Faculty of Medicine, University of Malaya, Kuala Lumpur, Malaysia

Correspondence: Dr Mei-Ling Sharon Tai, Consultant, Division of Neurology, Department of Medicine, Faculty of Medicine, University of Malaya, Kuala Lumpur, Malaysia. sharont1990@gmail.com 
scores, timing and imaging findings, were recorded. Vascular risk factors comprised diabetes mellitus (DM), hypertension, hyperlipidaemia, atrial fibrillation and smoking history. Stroke subtypes were categorised according to the TOAST (Trial of ORG 10172 in Acute Stroke Treatment) criteria. ${ }^{(11)}$

Eligible patients were administered with standard dose IV rt-PA $0.9 \mathrm{mg} / \mathrm{kg}$ (maximum $90 \mathrm{mg}$ ) within 4.5 hours of symptom onset, based on United States Food and Drug Administrationapproved indications. ${ }^{(12)} 10 \%$ was administered intravenously as a bolus dose and the remaining dose was given by continuous infusion over one hour. NIHSS scores were recorded on admission and discharge. The clinical course and progress was documented. The mRS was used to evaluate the clinical outcome at three months. ${ }^{(13)}$ An mRS score of 0-2 was classified as a good clinical outcome, whereas a score of 3-6 was categorised as a poor clinical outcome. An assessment of the THRIVE score was also made on admission, as the baseline score. ${ }^{(14)}$ In this study, a THRIVE score of 0-2 was defined as a good prognosis and 3-5 was defined as a moderately good prognosis. A score of 6-9 was defined as a poor prognosis.

Haemorrhagic transformation post thrombolysis was graded according to the ECASS I (European Cooperative Acute Stroke Study I) criteria. ${ }^{(15)}$ Symptomatic intracerebral haemorrhage $(\mathrm{SICH})$ was defined as the presence of intracerebral haemorrhage with worsening of NIHSS score by $>4$ points occurring within 48 hours of administration of rt-PA. ${ }^{(15)}$ Delayed haemorrhage was defined as intracerebral haemorrhage occurring $\geq 48$ hours after the administration of rt-PA. ${ }^{(16)}$ Mortality due to any cause was also recorded.

Data was analysed using IBM SPSS Statistics version 19.0 (IBM Corp, Armonk, NY, USA). Numerical variables were expressed as median, mean \pm standard deviation and range. Categorical variables of patients' baseline characteristics were presented as percentages. Continuous variables were analysed using the independent Student's t-test. Chi-square (or Fisher's exact test) was used to analyse the association between functional outcome and vascular risk factors. Multiple logistic regression analysis was performed to determine the predictors of functional outcome, and included variables that were statistically significant on univariate analysis. A two-tailed p-value $<0.05$ was considered to be statistically significant.

\section{RESULTS}

The demographic characteristics and stroke subtypes are shown in Table I. During the study period, 38 patients received IV rt-PA. Two patients, who had an mRS score of 2 and 3 on discharge, were lost to follow-up at three months and were excluded from the study. A total of 400 patients with ischaemic stroke were admitted to the neurology ward annually. During the study period, 1,800 ischaemic stroke patients were admitted to the hospital. Hence, only $2.0 \%$ of these patients were treated with IV rt-PA.

The median age of our study population was 59.0 (range $37-79)$ years, and $75.0 \%$ were men. Hypertension (83.3\%) was the most common risk factor. The median NIHSS score was 13.5
Table I. Baseline characteristics of patients treated with intravenous rt-PA $(n=36)$.

\begin{tabular}{|ll|}
\hline Characteristic & No. (\%) \\
\hline Age* (yr) & $59.11 \pm 10.47(59.0)$ \\
\hline Gender & \\
\hline Male & $27(75.0)$ \\
\hline Female & $9(25.0)$ \\
\hline Ethnicity & \\
\hline Malay & $21(58.3)$ \\
\hline Chinese & $8(22.2)$ \\
\hline Indian & $7(19.4)$ \\
\hline Hypertension & $30(83.3)$ \\
\hline Diabetes mellitus & $14(38.9)$ \\
\hline Hyperlipidaemia & $25(69.4)$ \\
\hline History of smoking & $16(44.4)$ \\
\hline Atrial fibrillation & $7(19.4)$ \\
\hline Ischaemic heart disease & $9(25.0)$ \\
\hline NIHSS score pre rt-PA* & $14.11 \pm 6.08(13.5)$ \\
\hline SBP pre rt-PA* (mmHg) & $150.44 \pm 20.60(148.5)$ \\
\hline Onset-to-treatment time* & $211.08 \pm 39.00(210.0)$ \\
\hline TOAST stroke subtypes & \\
\hline Large artery atherosclerosis & $26(72.2)$ \\
\hline Cardioembolism & $9(25.0)$ \\
\hline $\begin{array}{l}\text { Lacunar stroke secondary to small } \\
\text { vessel disease }\end{array}$ & $1(2.8)$ \\
\hline Modified Rankin scale score at 3 mth & \\
\hline 0-1 & $16(44.4)$ \\
\hline 0-2 & $20(55.6)$ \\
\hline SICH & $2(5.6)$ \\
\hline Delayed intracerebral haemorrhage & $5(13.9)$ \\
\hline THRIVE score* & $3.53 \pm 1.75(3.0)$ \\
\hline 0-2 & $10(27.8)$ \\
\hline $3-5$ & $21(58.3)$ \\
\hline 6-9 & $5(13.9)$ \\
\hline Mortality & \\
\hline NIHSS score on admission* & \\
\hline NIHSS score on discharge* & \\
\hline Data & \\
\hline
\end{tabular}

*Data presented as mean \pm standard deviation (median). NIHSS: National Institutes of Health Stroke Scale; rt-PA: thrombolysis with alteplase; THRIVE: Totaled Health Risks in Vascular Events; TOAST: Trial of ORG 10172 in Acute Stroke Treatment; SBP: systolic blood pressure; SICH: symptomatic intracerebral haemorrhage

(range 4-30) on admission and 6.5 (0-27) on discharge. Large artery atherosclerosis (72.2\%) was the most common stroke subtype. The stroke territories involved were areas supplied by the middle cerebral artery $(77.8 \%)$, middle cerebral artery with anterior cerebral artery (5.5\%), basilar artery $(5.5 \%)$, posterior cerebral artery $(2.8 \%)$, posterior inferior cerebellar artery $(2.8 \%)$ and lacunar infarcts (5.5\%). The median dose of rt-PA was 63.0 (range 42-90) mg, median onset-to-treatment time was 210.0 (range 120-270) minutes, and median systolic blood pressure before administration of rt-PA was 148.5 (range 102-199) $\mathrm{mmHg}$. All the patients had blood pressure below the target of 185/110 $\mathrm{mmHg}$ before rt-PA was initiated. 
Table II. Univariate analysis of factors determining functional outcome.

\begin{tabular}{|c|c|c|c|}
\hline \multirow[t]{2}{*}{ Parameter } & \multicolumn{2}{|c|}{ No. (\%) } & \multirow[t]{2}{*}{ p-value } \\
\hline & Good outcome $(n=20)$ & Poor outcome $(n=16)$ & \\
\hline Age* $^{*}(y r)$ & $58.40 \pm 11.18$ & $60.00 \pm 9.79$ & 0.66 \\
\hline Gender & & & 0.47 \\
\hline Male & $16(80.0)$ & $11(68.8)$ & \\
\hline Female & $4(20.0)$ & $5(31.3)$ & \\
\hline Ethnicity & & & 1.00 \\
\hline Malay & $12(60.0)$ & $9(56.3)$ & \\
\hline Chinese & $4(20.0)$ & $4(25.0)$ & \\
\hline Indian & $4(20.0)$ & $3(18.8)$ & \\
\hline NIHSS score pre rt-PA* & $12.05 \pm 4.79$ & $16.69 \pm 6.66$ & 0.03 \\
\hline Hypertension & & & 0.67 \\
\hline Yes & $16(80.0)$ & $14(87.5)$ & \\
\hline No & $4(20.0)$ & $2(12.5)$ & \\
\hline Diabetes mellitus & & & 0.06 \\
\hline Yes & $5(25.0)$ & $9(56.3)$ & \\
\hline No & $15(75.0)$ & $7(43.8)$ & \\
\hline Hyperlipidaemia & & & 0.16 \\
\hline Yes & $16(80.0)$ & $9(56.3)$ & \\
\hline No & $4(20.0)$ & $7(43.8)$ & \\
\hline History of smoking & & & 0.45 \\
\hline Yes & $10(50.0)$ & $6(37.5)$ & \\
\hline No & $10(50.0)$ & $10(62.5)$ & \\
\hline Atrial fibrillation & & & 0.21 \\
\hline Yes & $2(10.0)$ & $5(31.3)$ & \\
\hline No & $18(90.0)$ & $11(68.8)$ & \\
\hline Ischaemic heart disease & & & 0.15 \\
\hline Yes & $3(15.0)$ & $6(37.5)$ & \\
\hline No & $17(85.0)$ & $10(62.5)$ & \\
\hline Onset-to-treatment time* (min) & $208.20 \pm 40.96$ & $214.69 \pm 37.39$ & 0.63 \\
\hline Stroke subtype & & & 0.049 \\
\hline Cardioembolic & $2(10.0)$ & $7(43.8)$ & \\
\hline Non-cardioembolic & $18(90.0)$ & $9(56.3)$ & \\
\hline Blood glucose before thrombolysis* (mmol/L) & $9.10 \pm 3.56$ & $10.38 \pm 4.41$ & 0.34 \\
\hline THRIVE score* & $2.90 \pm 1.21$ & $4.31 \pm 2.02$ & 0.02 \\
\hline $0-2$ & $7(35.0)$ & $3(18.8)$ & \\
\hline $3-5$ & $13(65.0)$ & $8(50.0)$ & \\
\hline $6-9$ & $0(0)$ & $5(31.3)$ & \\
\hline Intracerebral haemorrhage $^{\dagger}$ & & & 0.21 \\
\hline Yes & $2(10.0)$ & $5(31.3)$ & \\
\hline No & $18(90.0)$ & $11(68.8)$ & \\
\hline
\end{tabular}

Modified Rankin scale score 0-2 was defined as a good outcome and 3-6 as a poor outcome. *Data presented as mean \pm standard deviation. †Symptomatic intracerebral haemorrhage and delayed haemorrhage. NIHSS: National Institutes of Health Stroke Scale; rt-PA: thrombolysis with alteplase; THRIVE: Totaled Health Risks in Vascular Events

Among the $2(5.6 \%)$ patients with $\mathrm{SICH}, 1$ (2.8\%) had parenchymal haematoma (PH) Type 2 and 1 (2.8\%) had haemorrhagic infarction (HI) Type 1. 5 (13.9\%) patients had delayed intracerebral haemorrhage; 2 (5.6\%) had PH Type 1, 2 (5.6\%) had PH Type 2 and 1 (2.8\%) patient had HI Type 1. The median $\mathrm{mRS}$ score at three months was 2 (range 0-6), with $16(44.4 \%)$ patients scoring $0-1$ and 20 (55.6\%) patients scoring 0-2. The median THRIVE score was 3.0 (range 0-7), with
$10(27.8 \%)$ patients scoring $0-2,21$ (58.3\%) patients scoring 3-5 and $5(13.9 \%)$ patients scoring 6-9 (Table I).

The in-hospital mortality rate was $11.1 \%(n=4)$. One patient died due to $\mathrm{SICH}$, while three other patients died secondary to acute coronary syndrome within the first seven days of hospitalisation. No other patient died within the first three months after discharge.

Table II shows the results of univariate analysis of good and poor functional outcomes. Compared to those with poor 
Table III. Multiple logistic regression of factors determining functional outcome.

\begin{tabular}{|lllll|}
\hline Parameter & $\boldsymbol{\beta}$ & $\mathbf{p}$-value & OR & $\mathbf{9 5 \%} \mathbf{C I}$ \\
\hline NIHSS score pre rt-PA & -0.371 & 0.032 & 0.69 & $0.49-0.97$ \\
\hline Diabetes mellitus & 3.682 & 0.010 & 40.00 & $2.38-662.31$ \\
\hline THRIVE score & 0.587 & 0.290 & 1.80 & $0.60-5.39$ \\
\hline Stroke subtype* & 1.689 & 0.160 & 5.41 & $0.51-57.87$ \\
\hline
\end{tabular}

${ }^{*}$ Cardioembolic vs. non-cardioembolic stroke. $\mathrm{Cl}$ : confidence interval NIHSS: National Institutes of Health Stroke Scale; OR: odds ratio; rt-PA thrombolysis with alteplase; THRIVE: Totaled Health Risks in Vascular Events

outcomes, the patients with good outcomes had a lower mean NIHSS score $(16.69 \pm 6.66$ vs. $12.05 \pm 4.79 ; \mathrm{p}=0.03)$ and a lower mean THRIVE score $(4.31 \pm 2.02$ vs. $2.90 \pm 1.21 ; p=0.02)$. Among the 20 patients with good outcomes, there was a higher proportion $(90.0 \%, \mathrm{n}=18)$ of patients with non-cardioembolic stroke (i.e, large artery disease and lacunar stroke), compared to $2(10.0 \%)$ patients with cardioembolic stroke $(p=0.049)$. In the group with good outcomes, there was also a higher proportion of patients without DM $(75.0 \%, \mathrm{n}=15)$ compared to only $25.0 \%$ $(n=5)$ of patients with DM $(p=0.06)$.

There was no statistically significant association between functional outcome and the risk factors of age, gender, ethnicity, hypertension, hyperlipidaemia, history of smoking, atrial fibrillation, ischaemic heart disease, onset-to-treatment time, blood glucose level before thrombolysis and presence of intracerebral haemorrhage on univariate analysis. Multiple logistic regression (Table III) showed that functional outcome had a statistically significant association with NIHSS score before rt-PA $(p=0.032)$ and DM $(p=0.010)$.

\section{DISCUSSION}

As stroke thrombolysis is a new and limited service in Malaysia, the data in this paper will be useful to help in the expansion of the current service. It could also assist in the development of similar services in other hospitals in Malaysia. Our findings show that stroke thrombolysis is safe, with similar outcomes in terms of functional status, $\mathrm{SICH}$ and mortality to those in the published literature. In our study, $44.4 \%$ of our patients had a three-month mRS score of 0-1 (i.e. excellent outcome), corresponding to other published data in Asia $(26.1 \%-59.0 \%) .{ }^{(17-26)}$ In the studies conducted in Europe and Canada, a three-month mRS score of $0-1$ was observed in $31 \%-53 \%$ of the patients. ${ }^{(27-30)} \mathrm{SICH}$ occurred in $5.6 \%$ of the patients, which was also similar to the previous literature. In studies in the Far East, $\mathrm{SICH}$ rates ranged from $3.1 \%$ to $7.0 \% .^{(24,25,31)}$ In Vietnam, $\mathrm{SICH}$ occurred in $5.5 \%$ of the patients. ${ }^{(20)}$

In this study, the three-month mortality rate of $11.1 \%$ was comparable with previous studies that reported three-month mortality rates of $6.5 \%-15.0 \% .^{(7,20,24,25,32-34)}$ However, our inhospital mortality rate was higher than the percentage reported in Singapore (3.7\%). ${ }^{(7,17)}$ Another multi-centre study had a one-week mortality rate of $1.5 \%$. ${ }^{(33)}$

The prognostic factors identified in this study were DM and baseline NIHSS score, a finding that was consistent with the published literature. ${ }^{(17,18,24,35-37)}$ The THRIVE score at baseline was associated with functional outcome at three months, proving its utility for prognostication in this cohort. There is presently limited data on THRIVE scoring among Asians. To our knowledge, this study is the second study on the evaluation of thrombolysed stroke patients in Asia with the THRIVE score. The first study in Asia evaluated the usefulness of the score in predicting prognosis in Chinese stroke patients treated with rt-PA. ${ }^{(38)}$

THRIVE score strongly predicts prognosis, with an increased score suggesting a higher risk of poor prognosis after stroke thrombolysis. ${ }^{(8,39,40)}$ It is superior to the other outcome scoring systems such as HIAT (Houston Intra-Arterial Therapy), HIAT-2, HAT (Haemorrhage After Thrombolysis) and SPAN-100 (Stroke Prognostication Using Age and NIHSS-100) in the prediction of prognosis and mortality ${ }^{(8)}$ Moreover, the THRIVE score is a good predictor of intracerebral haemorrhage after rt-PA administration at three months. ${ }^{(8,39,40)}$ The fact that it is simple to use also makes it a suitable instrument for developing Asian countries. ${ }^{(8)}$

The main strength of this study was the use of known measures and THRIVE scores, which allowed for comparison with the published literature. Its main limitation was the small sample size consisting of only patients from a single urban hospital, resulting in our statistical results being less than robust. The small sample size was due to transportation challenges and the health-seeking behaviour of the patients in Kuala Lumpur, Malaysia.

In conclusion, in our newly established service, IV rt-PA was safe with a $\mathrm{SICH}$ rate of $5.6 \%$ and had similar functional outcomes compared to the literature from this region. Poor functional outcome was associated with higher baseline NIHSS scores and the presence of DM. The THRIVE score was predictive of patients' final functional status.

\section{REFERENCES}

1. Facts and Figures. In: World Stroke Campaign [online]. Available at: http:// www.worldstrokecampaign.org/learn/facts-and-figures.html. Accessed October 9, 2016.

2. Loo KW, Gan SH. Burden of stroke in Malaysia. Int J Stroke 2012; 7:165-7.

3. Yusoff UN, Mahat D, Omar A, et al. Burden of premature mortality in Malaysia. Int J Public Health Res 2013; 3:249-56.

4. Ministry of Health, Malaysia. Health facts 2016. Available at: http://www.moh. gov.my/images/gallery/publications/KKM\%20HEALTH\%20FACTS\%202016. pdf. Accessed October 9, 2016.

5. Department of Statistics, Malaysia. Population distribution and basic demographic characteristic report 2010. Available at: https:// www.dosm.gov.my/v1/index.php?r=column/ctheme\&menu_

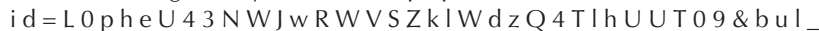
id=MDMxdHZjWTk1SjFzTzNkRXYzcVZjdz09. Accessed October 9, 2016.

6. van der Worp HB, van Gijn J. Clinical practice. Acute ischaemic stroke. N Engl J Med 2007; 357:572-9.

7. Sharma VK, Tsivgoulis G, Teoh HL, Ong BK, Chan BP. Stroke risk factors and outcomes among various Asian ethnic groups in Singapore. J Stroke Cerebrovasc Dis 2012; 21:299-304.

8. Flint AC, Faigeles BS, Cullen SP, et al; VISTA Collaboration. THRIVE score predicts ischemic stroke outcomes and thrombolytic hemorrhage risk in VISTA. Stroke 2013; 44:3365-9.

9. The THRIVE score. Available at: http://www.thrivescore.org. Accessed October 9, 2016.

10. National Institute of Neurological Disorders and Stroke rt-PA Stroke Study Group. Tissue plasminogen activator for acute ischemic stroke. N Engl J Med 1995; 333:1581-7.

11. Adams HP Jr, Bendixen BH, Kappelle LJ, et al. Classification of subtype of acute ischemic stroke. Definitions for use in a multicenter clinical trial. TOAST. Trial of Org 10172 in Acute Stroke Treatment. Stroke 1993; 24:35-41.

12. Parker S, Ali Y. Changing contraindications for t-PA in acute stroke: review of 
20 years since NINDS. Curr Cardiol Rep 2015; 17:81.

13. Nunn A, Bath PM, Gray LJ. Analysis of the Modified Rankin Scale in randomized controlled trials of acute ischemic stroke: a systematic review. Stroke Res Treat 2016; 2016:9482876.

14. Flint AC, Cullen SP, Faigeles BS, Rao VA. Predicting long-term outcome after endovascular stroke treatment: the totaled health risks in vascular events score. AJNR Am J Neuroradiol 2010; 31:1192-6.

15. Fiorelli M, Bastianello S, von Kummer R, et al. Hemorrhagic transformation within 36 hours of a cerebral infarct: relationships with early clinica deterioration and 3-month outcome in the European Cooperative Acute Stroke Study I (ECASS I) cohort. Stroke 1999; 30:2280-4

16. Derex L, Nighoghossian N. Intracerebral haemorrhage after thrombolysis for acute ischaemic stroke: an update. J Neurol Neurosurg Psychiatry 2008 79:1093-9.

17. Sharma VK, Tsivgoulis G, Tan JH, et al. Feasibility and safety of intravenous thrombolysis in multiethnic Asian stroke patients in Singapore. J Stroke Cerebrovasc Dis 2010; 19:424-30.

18. Yeo LL, Paliwal P, Teoh HL, et al. Early and continuous neurologic improvements after intravenous thrombolysis are strong predictors of favourable long-term outcomes in acute ischemic stroke. I Stroke Cerebrovasc Dis 2013; 22:e590-6.

19. Yeo LL, Paliwal P, Teoh HL, et al. Timing of recanalization after intravenous thrombolysis and functional outcomes after acute ischemic stroke. JAMA Neurol 2013; 70:353-8.

20. Nguyen TH, Truong AL, Ngo MB, et al. Patients with thrombolysed stroke in Vietnam have an excellent outcome: results from the Vietnam Thrombolysis Registry. Eur J Neurol 2010; 17:1188-92.

21. Nilanont Y, Nidhinandana S, Suwanwela NC, et al; Thai Stroke Registry. Quality of acute ischemic stroke care in Thailand: a prospective multicenter countrywide cohort study. J Stroke Cerebrovasc Dis 2014; 23:213-9.

22. Wang HR, Chen M, Wang FL, et al. Comparison of therapeutic effect of recombinant tissue plasminogen activator by treatment time after onset of acute ischemic stroke. Sci Rep 2015; 5:11743.

23. Pan SM, Liu JF, Liu M, et al. Efficacy and safety of a modified intravenous recombinant tissue plasminogen activator regimen in Chinese patients with acute ischemic stroke. J Stroke Cerebrovasc Dis 2013; 22:690-3.

24. Wong EH, Lau AY, Soo YO, et al. Is stroke thrombolysis safe and efficacious in Hong Kong? Hong Kong Med J 2012; 18:92-8.

25. Kim BJ, Han MK, Park TH, et al. Low-versus standard-dose alteplase for ischemic strokes within 4.5 hours: a comparative effectiveness and safety study. Stroke 2015; 46:2541-8.

26. Khurana D, Das B, Kumar A, et al. Temporal trends in intravenous thrombolysis in acute ischemic stroke: experience from a tertiary care center in India. J Stroke Cerebrovasc Dis 2017; 26:1266-73.

27. Willeit J, Geley T, Schöch J, et al. Thrombolysis and clinical outcome in patient with stroke after implementation of the Tyrol Stroke Pathway: a retrospective observational study. Lancet Neurol 2015; 14:48-56.

28. Machado C, Pinho J, Alves JN, et al. Five-year outcome in stroke patients submitted to thrombolysis. Stroke 2015; 46:2312-4.

29. Shobha N, Buchan AM, Hill MD; Canadian Alteplase for Stroke Effectiveness Study (CASES). Thrombolysis at 3-4.5 hours after acute ischemic stroke onset-evidence from the Canadian Alteplase for Stroke Effectiveness Study (CASES) registry. Cerebrovasc Dis 2011; 31:223-8.

30. Walters MR, Muir KW, Harbison J, Lees KR, Ford GA. Intravenous thrombolysis for acute ischaemic stroke: preliminary experience with recombinant tissue plasminogen activator in the UK. Cerebrovasc Dis 2005; 20:438-42.

31. Liao X, Wang Y, Pan Y, et al; Thrombolysis Implementation and Monitor of Acute Ischemic Stroke in China Investigators. Standard-dose intravenous tissuetype plasminogen activator for stroke is better than low doses. Stroke 2014; 45:2354-8

32. Salam KA, Ummer K, Pradeep Kumar VG, Noone ML. Intravenous thrombolysis for acute ischemic stroke in the 3- to 4.5-hour window--the Malabar experience. Int J Stroke 2014; 9:426-8.

33. Anderson CS, Robinson T, Lindley RI,et al; ENCHANTED Investigators and Coordinators. Low-dose versus standard-dose intravenous alteplase in acute ischemic stroke. N Engl J Med 2016; 374:2313-23.

34. Nakagawara J, Minematsu K, Okada Y, et al; J-MARS Investigators. Thrombolysis with $0.6 \mathrm{mg} / \mathrm{kg}$ intravenous alteplase for acute ischemic stroke in routine clinical practice: the Japan post-Marketing Alteplase Registration Study (J-MARS). Stroke 2010; 41:1984-9.

35. Endo K, Koga M, Sakai N, et al; Joint Research Group from JR-NET2 and SAMURAI Study Investigators. Stroke outcomes of Japanese patients with major cerebral artery occlusion in the post-alteplase, pre-MERCI era. J Stroke Cerebrovasc Dis 2013; 22:805-10.

36. Bhardwaj A, Sharma G, Raina SK, Sharma A, Angra M. Advanced age and higher national institutes of health stroke scale score as predictors of poor outcome in ischemic stroke patients treated with alteplase: a study from a tertiary care centre in rural north-west India. J Neurosci Rural Pract 2017; 8:236-40.

37. Yoneda Y, Yamamoto S, Hara Y, et al. Post-licensed 1-year experience of systemic thrombolysis with tissue plasminogen activator for ischemic stroke in a Japanese neuro-unit. Clin Neurol Neurosurg 2007; 109:567-70.

38. Chen W, Pan Y, Zhao X, et al. Totaled health risks in vascular events score predicts clinical outcome and symptomatic intracranial hemorrhage in Chinese patients after thrombolysis. Stroke 2015; 46:864-6.

39. You S, Han Q, Xiao G, et al. [The role of THRIVE score in prediction of outcomes of acute ischemic stroke patients with atrial fibrillation]. Zhonghua Nei Ke Za Zhi 2014; 53:532-6. Chinese.

40. Runde D. THRIVE Score for Stroke Outcome. Available at: http://www.mdcalc. com/thrive-score-for-stroke-outcome/. Accessed October 9, 2016. 